(c) 2019 Universidad Nacional Autónoma de México, Facultad de Estudios Superiores Zaragoza.

Este es un artículo Open Access bajo la licencia CC BY-NC-ND (http://creativecommons.org/licenses/by-nc-nd/4.0/).

TIP Revista Especializada en Ciencias Químico-Biológicas, 22: 1-8, 2019.

DOI: 10.22201/fesz.23958723e.2019.0.193

\title{
Lagos como sensores de cambio climático: el caso de La Alberca de Tacámbaro, Michoacán, México
}

\author{
Margarita Caballero ${ }^{1}$ y Gabriela Vázquez ${ }^{2}$ \\ ${ }^{1}$ Laboratorio de Paleolimnología, Instituto de Geofísica, Universidad Nacional Autónoma \\ de México, Coyoacán 04510, Ciudad de México, México. ${ }^{2}$ Instituto de Ecología, A.C., \\ Carretera Antigua a Coatepec \# 351, el Haya, 91073, Xalapa, Ver., México. \\ E-mail:maga@igeofisica.unam.mx
}

\begin{abstract}
RESUMEN
Los lagos están entre los ecosistemas más sensibles al cambio climático, ya que el comportamiento termodinámico de su columna de agua es controlado por la marcha anual de la temperatura. Los lagos tropicales profundos presentan una columna de agua estratificada durante los meses más cálidos, sin embargo, en invierno esos estratos desaparecen al mezclarse la columna de agua. El patrón de mezcla de un lago se puede alterar cuando se presenta un incremento en la temperatura, ya sea debido al calentamiento global y/o durante el fenómeno meteorológico conocido como "El Niño". El objetivo de este trabajo fue correlacionar cambios en el clima con los del lago La Alberca de Tacámbaro, Michoacán, México, durante un período que involucra dos eventos de "El Niño" (2009-2010 y 2015-2016) y con variaciones en las asociaciones de diatomeas preservadas en los sedimentos entre 1988 a 2015. El lago presentó un patrón de mezcla en invierno, con una termoclina somera en primavera que se profundiza en el otoño. Los datos muestran una tendencia que se refleja en la limitación de dos nutrientes, fósforo y nitrógeno (P y N), asociada con una menor mezcla vertical causada por años más calientes desde el 2000 y el efecto de los años en los que se presenta el fenómeno "El Niño". Estos cambios correlacionan con un calentamiento del hipolimnion en 2015 comparado con 2009 y con alteraciones en la diversidad y composición de las diatomeas entre 1988 a 2015. Este estudio permitió documentar los cambios recientes del lago y asociarlos con el impacto del calentamiento global y los eventos de "El Niño".

Palabras Clave: lagos tropicales, Limnología, calentamiento global, diatomeas, "El Niño", Michoacán.
\end{abstract}

Lakes as climate change sensors: the case of La Alberca de Tacámbaro, Michoacán, México

\begin{abstract}
Lakes are excellent sensors of climate changes because their thermal behavior is controlled by the annual temperature patterns. Deep tropical lakes stratify during the warm part of the year, mixing during winter. This mixing pattern can be altered by warmer climatic conditions such as global warming and/or "El Niño". In this article we aim to correlate climate variability with changes in the mixing pattern of lake Alberca de Tacámbaro, Michoacán, México during an interval that involved two "El Niño" events (2009-2010 and 2015-2016) and with changes in the diatom assemblages preserved in the sediments between 1988 to 2015. The lake showed a winter mixing pattern, with a shallower thermocline in spring that deepened towards the autumn. Temperature data show a warming trend since AD 2000 and the impact "El Niño" years is associated to nutrient limitation (phosphorus and nitrogen), that we associate to a reduced vertical mixing. These changes are linked with an increase in hypolimnion temperature in 2015 compared to 2019 and modifications in the diversity and species composition of the diatom community of this lake between 1988 to 2015. This study allowed to identify recent changes in the lake and its diatom assemblages that are linked with global warming trends and "El Niño" events.
\end{abstract}

Key Words: tropical lakes, Limnology, Global Warming, diatoms, ENSO, Michoacán.

Nota: Artículo recibido el 06 de septiembre del 2019 y aceptado el

20 de noviembre del 2019. 


\section{INTRODUCCIÓN}

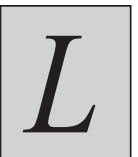

os lagos están entre los ecosistemas más sensibles al cambio climático, ya que el comportamiento termodinámico de su columna de agua es controlado fundamentalmente por la marcha anual de la temperatura. En latitudes tropicales como México, los lagos relativamente profundos $(>10 \mathrm{~m})$, presentan un patrón de mezcla conocido como cálido monomíctico (Lewis, 2000), porque durante el invierno la columna de agua tiene una temperatura homogénea que permite la mezcla vertical, pero durante la primavera y el verano la capa superficial del agua se calienta, diferenciándose del agua profunda, formando dos estratos con temperaturas diferentes. El estrato cálido superficial (o epilimnion), se separa del estrato más frío y profundo (o hipolimnion) por una capa que presenta un intenso gradiente de temperatura a la que se denomina termoclina (o metalimnion), que efectivamente limita la mezcla vertical de la columna de agua. El hipolimnion, al quedar aislado de la atmósfera y de la luz solar, puede desarrollar anoxia, al consumirse el oxígeno por respiración y descomposición de la materia orgánica exportada por gravedad desde el epilimnion. El epilimnion por su parte, al recibir radiación solar, puede sostener niveles altos de productividad primaria, siempre y cuando existan suficientes nutrientes, principalmente nitrógeno y fósforo ( $\mathrm{N}$ y $\mathrm{P}$ ), pero éstos tienden a agotarse si no hay una mezcla vertical que los recicle desde el fondo del lago (hipolimnion). Durante el invierno, el epilimnion gradualmente se enfría y su temperatura llega a igualarse con la del hipolimnion, desapareciendo la estratificación y posibilitando la mezcla de la columna de agua, la recirculación de nutrientes hacia las capas más someras y el aporte de oxígeno a las capas más profundas.

El calentamiento global es un fenómeno que ha empezado a tener efectos medibles en los patrones de estratificación de algunos lagos, por ejemplo, se ha detectado una tendencia de la termoclina a establecerse en profundidades menores (somerización de la termoclina) y a que la estratificación presente una mayor estabilidad y duración, lo que lleva a una reducción en la mezcla vertical y una intensificación de la limitación de nutrientes en el epilimnion (ej. Shimoda et al., 2011, Winder, Reuter \& Schladow, 2009). Los cambios en el patrón de mezcla de los lagos afectan de manera directa e indirecta la composición, diversidad y sucesión anual del fitoplancton que habita en su columna de agua, lo que a su vez afecta la estructura del resto de la comunidad en niveles tróficos superiores. Se ha documentado que estos cambios han favorecido a especies más pequeñas del fitoplancton, lo que a su vez afecta las poblaciones de otros organismos como los peces, con un posible impacto social y económico (Catalan et al., 2013; Ndebele Murisa, Musil \& Raitt, 2010).

Los cambios reportados en los patrones de mezcla de los lagos asociados con el calentamiento global pueden exacerbarse en años particularmente cálidos, como pueden ser los años del fenómeno "El Niño" (Shimoda et al., 2011; Katz, Hampton, Izmest'eva \& Moore, 2011). Sin embargo, es muy difícil evaluar la intensidad de estos cambios (en el clima y los ecosistemas) en países como México, donde no hay una tradición de monitoreo ambiental que permita conocer las condiciones originales (base) de los ecosistemas y/o sus variaciones recientes. Bajo estas circunstancias, el enfoque paleolimnológico puede ayudar a poner en perspectiva los datos de monitoreos modernos y permitir su comparación con tendencias de cambio en los ecosistemas de más largo plazo, registradas en los sedimentos lacustres. El enfoque paleolimnológico involucra el análisis de algún indicador paleoambiental preservado en una secuencia sedimentaria mediante el cual se reconstruye la historia del lago y su entorno. Este enfoque es posible dado que en los lagos continuamente se acumulan sedimentos en el fondo, donde se concentran los restos de los organismos que viven en la columna de agua. Si estos restos son resistentes a la degradación, como ocurre con las valvas silíceas de las algas diatomeas (Bacillariophyceae), es posible estudiarlos estratigráficamente identificando los momentos de un cambio climático y/o ambiental como eventos de cambio en la abundancia o composición de las especies preservadas. Las diatomeas son particularmente buenos indicadores ambientales por ser abundantes, con historias de vida cortas, y porque su taxonomía se basa en gran medida en la estructura y morfología de su valva silícea que se preserva en los sedimentos.

En este trabajo analizamos datos meteorológicos entre 1988 a 2011 y los asociamos con datos obtenidos del análisis de diatomeas en una secuencia sedimentaria que abarca de 1988 a 2006 y con monitoreos modernos de sedimentos y columna de agua realizados entre 2009 y 2015 en el lago Alberca de Tacámbaro, Michoacán. Mediante estos análisis es posible documentar los cambios recientes ocurridos en este lago que asociamos con el del calentamiento global y los eventos del fenómeno meteorológico conocido como "El Niño".

\section{MATERIALES Y MÉTODOS}

La Alberca de Tacámbaro, Michoacán (19¹3’30” N, $101^{\circ} 27^{\prime} 30^{\prime}$ ' O, 1,460 m snm), es un lago cráter ubicado en el límite sur de la Faja Volcánica Trans-Mexicana (FVTM, Figura 1). Su clima es templado, con una temperatura media anual de $19{ }^{\circ} \mathrm{C}$ y una precipitación de 1,164 mm anuales, concentrada entre junio y octubre. Es un lago pequeño y relativamente profundo $(28 \mathrm{~m})$, ubicado dentro de un cráter con paredes empinadas, por lo que tiene una estrecha zona litoral. Esta morfología lo hace particularmente sensible al cambio ambiental, ya que al encontrarse protegido y tener una superficie relativamente pequeña, la mezcla de la columna de agua por arrastre del viento es mínima y su patrón de estratificación está controlado sólo por la marcha anual de la temperatura. Estudios previos realizados en La 
Alberca de Tacámbaro (Hernández-Morales, Ortega-Murillo, Alvarado-Villanueva, Sánchez-Heredia \& Medrano-Zarco, 2008; Hernández-Morales, Ortega-Murillo, Sánchez-Heredia, Alvarado-Villanueva \& Aguilera, 2011; Caballero, Vázquez, Ortega, Favila, Lozano-García, 2016), indican que es un lago ligeramente alcalino, eutrófico, de agua dulce, con un patrón de mezcla cálido monomíctico caracterizado por un breve periodo de mezcla en invierno (enero). Estos estudios han documentado que las algas clorofitas y las diatomeas son los grupos más abundantes y diversos dentro del fitoplancton de este lago. Además, las valvas silíceas de las diatomeas se preservan en los sedimentos de este lago, reflejando fielmente los cambios ocurridos en la composición de las especies en la columna de agua.

A partir de los datos mensuales de temperatura y precipitación entre 1930 y 2011 registrados por la estación Tacámbaro del Servicio Meteorológico Nacional (SMN) $\left(19^{\circ} 14^{\prime} \mathrm{N}\right.$, $101^{\circ} 28^{\prime}$ O, SMN-16,123), se calcularon las anomalías mensuales y anuales de temperatura y precipitación entre 1988 y 2011 (Figura 2). No fue posible realizar el análisis con datos climatológicos más recientes debido a que la serie de datos reportada por el SMN para esta estación sólo abarca hasta mayo de 2011. Por otro lado, se midieron perfiles de temperatura y oxígeno disuelto en el centro del lago La Alberca de Tacámbaro usando una sonda multiparamétrica (Hydrolab Quanta G) en ocho ocasiones durante invierno, primavera, verano y otoño de 2009 y 2015. En estas ocasiones también se tomaron muestras de agua con una botella Van Dorna a diferentes profundidades para realizar análisis de fósforo reactivo soluble (FRS) y de nitrógeno en forma de amoniaco, nitritos y nitratos. Las muestras se conservaron en refrigeración hasta ser analizadas en el laboratorio. Para estos análisis se siguieron los métodos analíticos descritos en Caballero et al. (2016).

Se realizaron análisis de diatomeas en los primeros $20 \mathrm{~cm}$ de una secuencia sedimentaria recuperada en 2006 con un nucleador de gravedad UWITEC (TAC0106GIII, $90 \mathrm{~cm}$ longitud total), tomando muestras de manera continua cada $1 \mathrm{~cm}$. Para contar con un control cronológico se seleccionó la muestra de $36 \mathrm{~cm}$ de profundidad para datación por

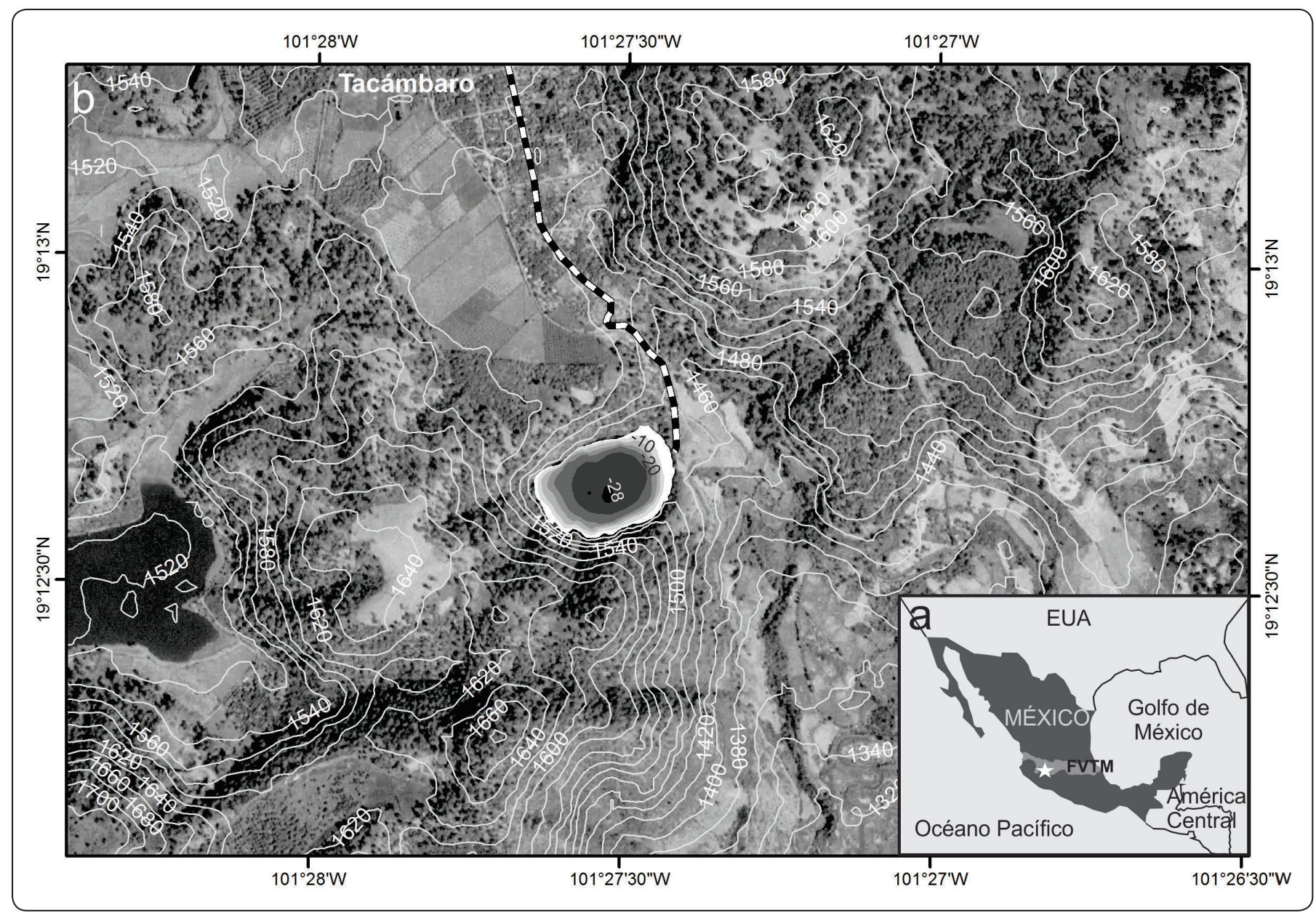

Figura 1. Mapa de localización del lago La Alberca de Tacámbaro, Michoacán. 
radiocarbono en un laboratorio comercial (Beta-324552: $126.1 \pm 0.5 \mathrm{pMC})$. Esta datación se calibró con el programa CALIBBomb (Hua, Barbetti \& Rakowski, 2013; Reimer et al., 2013) y se usó para generar un modelo de edad por interpolación polinomial entre el horizonte fechado y la cima de la secuencia (2006) (Caballero et al., 2016), lo que permite establecer que la sección de la secuencia estudiada data entre 1988 y 2006. Además, se colectaron muestras de sedimento superficial para análisis de diatomeas mediante una draga Ekman en agosto (verano) y octubre (otoño) de 2009, febrero (invierno) de 2010, junio (verano) de 2011, enero, abril, julio y noviembre (invierno, primavera, verano y otoño) de 2015. Por lo tanto, se cuenta con una serie de datos de diatomeas en sedimento que de manera conjunta abarca de 1988 a 2015.

Para los análisis de diatomeas se pesaron ente 0.2 y $0.5 \mathrm{~g}$ de sedimento seco, que se trataron con $\mathrm{HCl}(10 \%), \mathrm{H}_{2} \mathrm{O}_{2}$ y $\mathrm{HNO}_{3}$ para eliminar carbonatos y materia orgánica. Con $200 \mu \mathrm{L}$ de material limpio se montaron preparaciones permanentes usando Naphrax como medio de montaje. Se realizaron conteos de un mínimo de 400 valvas de diatomeas utilizando un microscopio Olympus BX50 (1000x) con contraste de fases interdiferencial, la identificación taxonómica se basó en literatura especializada (Krammer \& Lange-Bertalot 19861991). Los conteos se transformaron en concentraciones (valvas por gramo de sedimento seco), por el método de aproximación a la forma geométrica más cercana. Para cada muestra se calculó la riqueza específica $(S)$ y el índice de diversidad de Shannon $(H)$, además la diversidad verdadera o número efectivo de especies $\left({ }^{2} D\right)$ se calculó con la fórmula: $\left[{ }^{2} D=\right.$ exponencial $\left.(H)\right]$.

\section{RESUltados \\ Clima}

En la serie de datos climatológicos se observa un patrón en las anomalías de temperatura, con valores negativos antes del año 2000 , mientras que las anomalías positivas se registran después de esta fecha; este patrón refleja una clara tendencia reciente de calentamiento (Figura 2). En particular la mayor anomalía positiva del registro corresponde con la primavera-verano de 2009, un año de "El Niño". En los datos de precipitación no se observa ningún patrón de largo plazo, sin embargo, el año con la mayor anomalía positiva fue 2011, que corresponde con un año del fenómeno meteorológico conocido como "La Niña". Estas anomalías sugieren que el clima local es sensible a esta oscilación climática.

\section{Limnología}

Los perfiles de temperatura medidos en 2009 y en 2015 (Figura 3 ), muestran que en invierno (enero 2009 y 2015) la temperatura de la columna de agua era prácticamente homogénea, mientras que el resto de los meses el lago se encontraba estratificado, con un epilimnion e hipolimnion claramente diferenciados, confirmando un patrón de mezcla cálido monomíctico. A lo

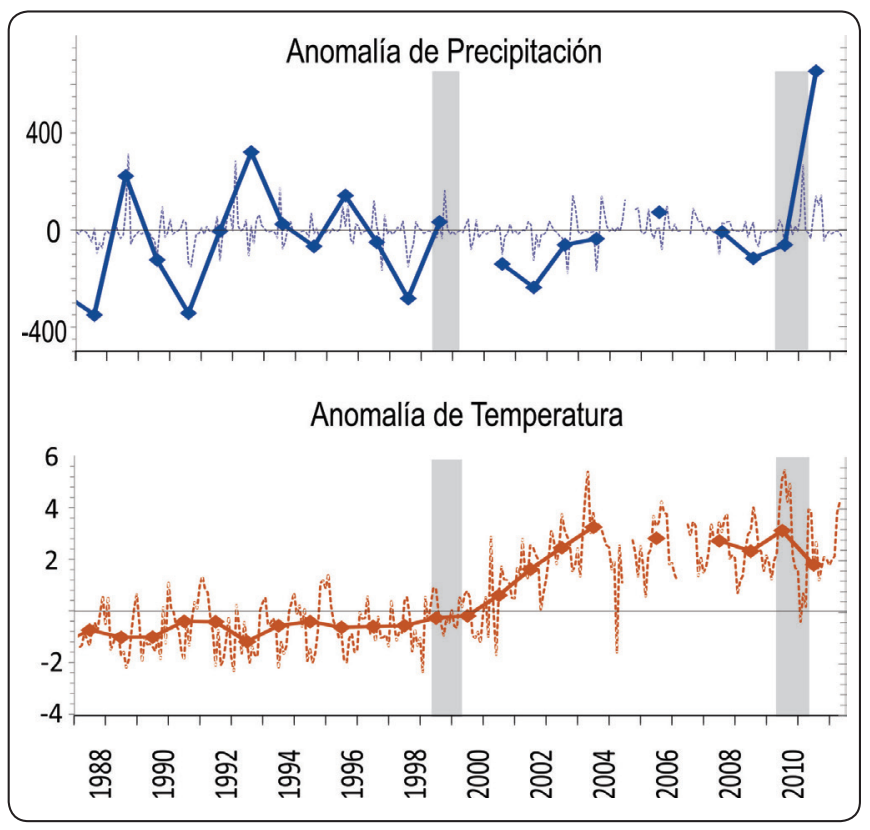

Figura 2. Anomalías de precipitación y temperatura con respecto a la media entre 1930-2011 en Tacámbaro, Michoacán (estación Tacámbaro: 16,123, Servicio Meteorológico Nacional). Las líneas continuas representan valores anuales mientras que las punteadas son valores mensuales.

largo del ciclo anual la termoclina varía en su profundidad, es más somera en primavera (ej. primavera 2015, 3 a $4 \mathrm{~m}$ ) y gradualmente se profundiza (ej. otoño 2015, 9 a $11 \mathrm{~m}$ ). El mayor gradiente térmico (diferencia de temperatura entre epilimnion e hipolimnion), se establece durante los meses de verano (2009 y 2015), siendo del orden de $8{ }^{\circ} \mathrm{C}$. Es interesante notar que la serie de datos de 2015 presentó temperaturas, en el hipolimnion, que son consistentemente mayores $\left(\sim 0.7^{\circ} \mathrm{C}\right)$ que durante 2009. Los perfiles de oxígeno disuelto (Figura 3), indican que las aguas profundas del lago $(>11 \mathrm{~m})$ permanecen anóxicas todo el año. Estos perfiles también muestran una oxiclina evidente en todos los muestreos, si bien menos desarrollada en enero de 2015, cuando las concentraciones de oxígeno en el epilimnion son las menores. La presencia de una oxiclina aún en invierno sugiere que la mezcla vertical en estos años (2009 y 2015), no fue lo suficientemente intensa para homogenizar químicamente la columna de agua. La oxiclina también muestra variaciones en su profundidad a lo largo del año, siendo más somera en invierno y primavera (ej. invierno 2015, 3-5 m) y más profunda en otoño (ej. otoño $2015,7.5-12 \mathrm{~m})$.

Las concentraciones de fósforo soluble reactivo (FSR) y de nitrógeno inorgánico disuelto (NID, la suma de amoniaco, nitritos y nitratos) en muestras de agua de distintas profundidades se presentan en la Figura 4; en esta figura se identifican fácilmente las muestras cuyas concentraciones de FSR y NID caen por debajo de $0.1 \mu \mathrm{M}$ y $7 \mu \mathrm{M}$ respectivamente, 


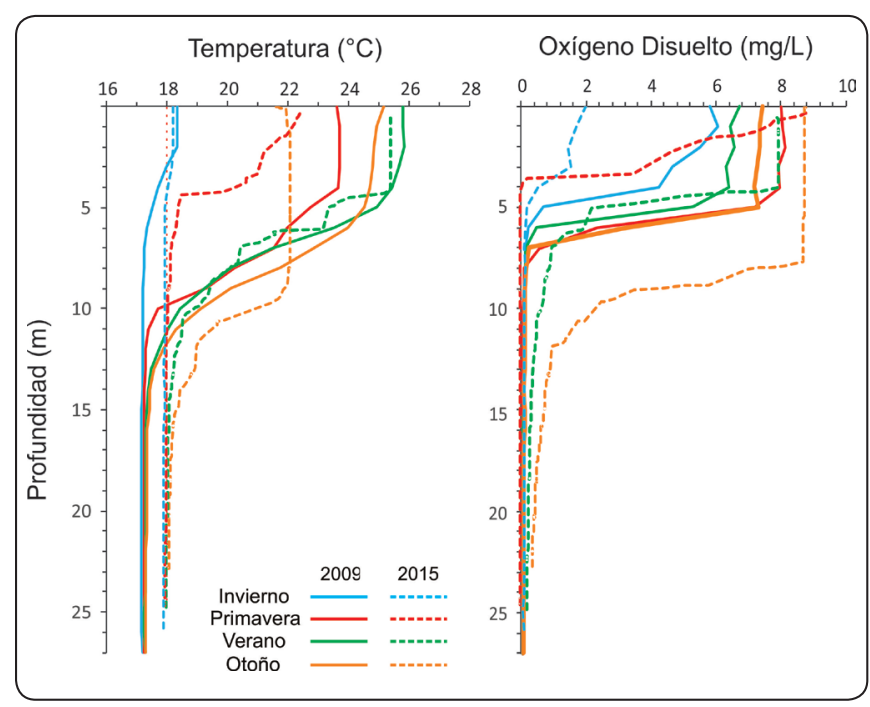

Figura 3. Perfiles de temperatura y oxígeno disuelto en una columna de agua a lo largo de dos ciclos anuales (2009 y 2015) en el lago La Alberca de Tacámbaro, Michoacán.

considerados valores umbrales por debajo de los que hay una limitación de la productividad primaria por alguno de estos nutrientes (Reynolds, 1999); en la misma Figura también se puede apreciar cuando la relación $\mathrm{N}: \mathrm{P}$ cae por arriba de (P-limitado) o por debajo de (N-limitado) la relación de Redfield de 16N:1P (Redfield, 1958). En la mayoría de las muestras de La Alberca de Tacámbaro la relación N:P es alta $(>16 \mathrm{~N}: 1 \mathrm{P})$, indicativo de que la productividad primaria en el lago por lo general es limitada por fósforo, como es el caso en las muestras de aguas relativamente someras $(<11 \mathrm{~m})$, de primavera y verano del 2009 y de invierno y primavera de 2015, que están por debajo del valor umbral de este nutriente. Por otra parte, las muestras someras $(<11 \mathrm{~m})$ de primavera y otoño 2009 están por debajo del umbral del nitrógeno, de tal forma que el epilimnion de primavera de 2009 se caracterizó por una co-limitación de ambos nutrientes, lo cual no ocurre en 2015.

\section{Paleolimnología}

En las muestras de sedimento estudiadas se registraron en total 34 taxones de diatomeas, con un promedio de 16 por muestra (rango de 11 a 21), de las cuales 10 fueron las más abundantes a lo largo del registro (Figura 2, Tabla I), siendo Discostella stelligera y $D$. pseudostelligera un complejo de especies difícil de distinguir bajo el microscopio de luz. La diversidad Shannon mostró un valor promedio de 1.2 (rango de 0.4 a 2), mientras que la diversidad verdadera (número efectivo de especies equitativamente distribuidas) en promedio fue de 3.7 (rango 1.5 a 7.1). La diversidad verdadera presentó valores bajos $(<3)$ antes de 2000 (Figura 5), cuando el conjunto de diatomeas estuvo dominado por Achnanthidium minutissimum en asociación con Aulacoseira granulata var. angustissima, Brachysira vitrea, Discostella stelligera/ D. pseudostelligera,

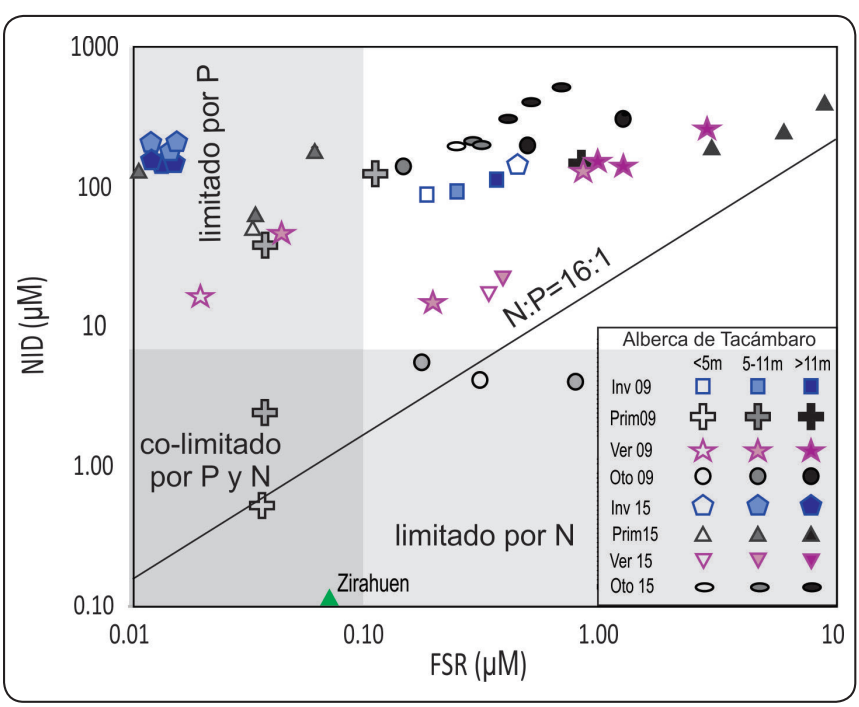

Figura 4. Concentraciones de fósforo soluble reactivo (FRS) y de nitrógeno inorgánico disuelto (NID) en muestras de agua de distintas profundidades a lo largo de dos ciclos anuales (2009 y 2015) en el lago La Alberca de Tacámbaro, Michoacán.

Achnanthidium minutissimum (Kützing) Czarnecki, 1994

Aulacoseira granulata var. angustissima (O. Müller)

Simonsen, 1979

Brachysira vitrea (Grunow) Ross, 1966

Discostella stelligera (Cleve \& Grunow) Houk \& Klee, 2004

Discostella pseudostelligera (Hustedt) Houk \& Klee, 2004

Fragilaria crotonensis Kitton, 1869

Fragilaria tenera var. nanana (Lange-Bertalot) LangeBertalot \& S.Ulrich, 2014

Nitzschia palea (Kützing) W. Smith, 1856

Pantocsekiella ocellata (Pantocsek) K.T. Kiss \& E. Ács et al., 2016

Ulnaria acus (Kützing) Aboal in Aboal et al., 2003

Ulnaria delicatissima (W. Smith) Aboal \& Silva, 2004

Tabla I. Listado taxonómico de las especies más abundantes en los sedimentos del lago La Alberca de Tacámbaro, Michoacán.

Fragilaria crotonensis y Ulnaria delicatissima. Después del año 2000 la diversidad verdadera aumentó, asociada con una disminución en las concentraciones de A. minutissimum y un incremento en especies que antes eran muy raras como Fragilaria tenera var. nanana o que no existían en el lago y como Pantocsekiella ocellata (Figura 5). Sólo la muestra de verano de 2011 tiene una diversidad muy baja, dominada por A. minutissimum. A diferencia de Pantocsekiella ocellata que aumenta su abundancia marcadamente en 2009 y nuevamente en 2015, cuando es una de las especies más abundantes, desplazando prácticamente a todas las demás con excepción de A. minutissimum. 


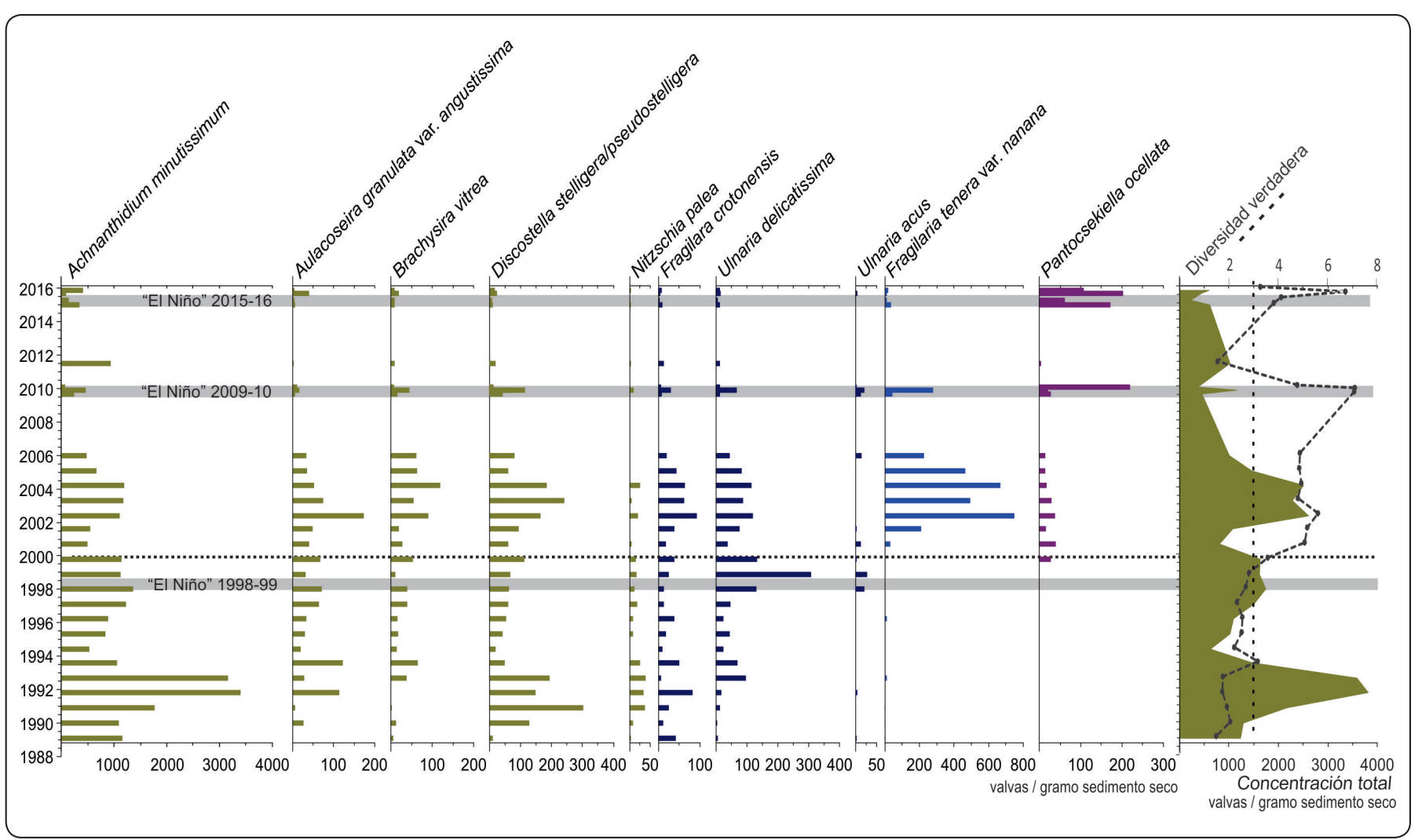

Figura 5. Asociaciones de diatomeas en los sedimentos de la secuencia sedimentaria TAC0106GIII y de dragas colectadas entre 2009 y 2015 en La Alberca de Tacámbaro, Michoacán.

\section{Discusión}

Las anomalías de temperatura en La Alberca de Tacámbaro indican una clara tendencia hacia años más calientes (anomalías positivas), después del año 2000 y en particular después de 2003. Esta tendencia de calentamiento es acorde con estudios realizados en México, que reportan una tendencia hacia mayores temperaturas en las décadas posteriores a 1970 (Pavia, Graef \& Reyes, 2009; Englehart \& Douglas, 2005). Estos datos también muestran que durante la primaveraverano del evento de "El Niño" de 2009-2010 se presentó una de las anomalías positivas más intensas del registro (Figura 2). Veranos más secos y cálidos han sido identificados como una de las anomalías climáticas que caracterizan a los años de "El Niño" en gran parte de México (Bravo Cabrera, Azpra Romero, Zarraluqui Such, Gary García \& Estrada Porrúa, 2010), por lo tanto, aunque no se cuenta con datos meteorológicos para la estación de Tacámbaro posteriores a 2011, es factible que durante el evento de "El Niño" de 2015, también se hayan presentado condiciones anómalas muy cálidas durante la primavera-verano de este año.

Uno de los efectos esperados del calentamiento global y con años anómalos muy cálidos como son los años de "El Niño", es que se reduzca la mezcla vertical y se incremente la limitación de nutrientes. Los datos de La Alberca de Tacámbaro sugieren que esto ocurrió durante los años estudiados, ya que tanto en enero de 2009 como de 2015 los perfiles de oxígeno mostraron una débil mezcla de invierno, que no logró homogeneizar químicamente la columna de agua, y los datos de NID y FRS mostraron limitación de nutrientes en las capas someras del lago tanto en primavera-verano de 2009 como en inviernoprimavera de 2015. Además de esto, entre 2009 y 2015 se registró un calentamiento en el hipolimnion del lago de aproximadamente $0.7^{\circ} \mathrm{C}$, aunque será necesario realizar más muestreos para confirmar que esta tendencia es permanente y no sólo una anomalía de corta duración.

El registro de las diatomeas de La Alberca de Tacámbaro presenta cambios importantes en la diversidad y composición de especies que se correlacionan tanto con el incremento en la temperatura después del año 2000 como con los eventos de "El Niño" 2009 y 2015 (y posiblemente 1998) y que muy posiblemente fueron favorecidos por la limitación de nutrientes. Antes de 2000 el conjunto de diatomeas es menos diverso y está dominado por Achnanthidium minutissimum en asociación con Aulacoseira granulata var. angustissima, Brachysira vitrea, Discostella stelligera/pseudostelligera, Fragilaria crotonensis y Ulnaria delicatissima. Sin embargo, 
con la tendencia hacia condiciones más cálidas después de 2000, el conjunto de diatomeas cambió, aumentando su diversidad al incrementarse la concentración de Fragilaria tenera var. nanana y aparecer en el registro Pantocsekiella ocellata. Esta última es una especie que es abundante en el lago de Zirahuén, Michoacán (triángulo verde en Figura 4), bajo condiciones de co-limitación de N y P (Bernal-Brooks, Dávalos-Lind \& Lind, 2002), por lo que consideramos que es una buena competidora bajo estas condiciones. Durante el evento de "El Niño" de 2009-2010 Pantocsekiella ocellata aumentó su concentración marcadamente y es probable que haya sido favorecida por la limitación de nutrientes en 2009, tanto en primavera ( $\mathrm{N}$ y $\mathrm{P}$ ) como en verano $(\mathrm{P})$. En verano de 2011 P. ocellata prácticamente desaparece del registro, pero regresa durante "El Niño" de 2015, cuando nuevamente existe limitación de nutrientes $(\mathrm{P})$ en invierno y primavera, desplazando a las otras especies, excepto a A. minutissimum.

\section{Conclusiones}

El lago La Alberca de Tacámbaro es un sistema acuático en el que se encontraron cambios importantes en los conjuntos de algas diatomeas que se pueden asociar con un aumento en la limitación de nutrientes durante el invierno y la primavera de años particularmente cálidos como son los años de "El Niño". También se pudieron documentar en este lago alteraciones importantes en la flora de diatomeas que se asocian con el calentamiento global registrado en los datos meteorológicos desde el año 2000, estas alteraciones involucran un cambio radical en la flora de diatomeas, con el establecimiento de una nueva especie dominante que es aparentemente favorecida por una mayor limitación de nutrientes, Pantocsekiella ocellata, la cual desplazó a casi todas las especies que eran abundantes con anterioridad. Consideramos que este tipo de investigación en sistemas acuáticos tropicales es muy importante para entender el impacto que los cambios climáticos y ambientales pueden tener sobre su biodiversidad y conservación.

\section{Agradecimientos}

Se agradece a la Coordinación General del Servicio Meteorológico Nacional (CGSMN) y a la Comisión Nacional del Agua (CONAGUA) por los datos meteorológicos proporcionados. Agradecemos también el apoyo técnico de Rosario Landgrave, Ariadna Martínez, Arlette Fuentes, Susana Sosa y Daniela Cela. Este estudio tuvo financiamiento de la UNAM-DGAPA proyectos PAPIIT IN101513 e IN100717.

\section{REFERENCIAS}

Bernal-Brooks, F.W., Dávalos-Lind, L. \& Lind, O.T. (2002.) Assessing trophic state of an endorheic tropical lake: the algal growth potential and limiting nutrients. Archiv. Für Hydrobiologie, 153, 323-338.

Bravo Cabrera, J.L., Azpra Romero, E., Zarraluqui Such, V., Gary García, C. \& Estrada Porrúa, F. (2010). Significance tests for the relationship between "El Niño" phenomenon and precipitation in Mexico. Geofisica Internacional, 49, 245-261.

Caballero, M., Vázquez, G., Ortega, B., Favila, M.E. \& Lozano-García, S. (2016). Responses to a warming trend and "El Niño" events in a tropical lake in western Mexico. Aquatic Sciences, 78, 591-604.

Catalan, J., Pla-Rabés, S., Wolfe, A., Smol, J., Rühland, K., Anderson, J. Kopáček, J., Stuchlík, E., Schmidt, R., Koinig, K., Camarero, L., Flower, R. \& Heiri, O. (2013). Global change revealed by paleolimnological records from remote lakes: a review. Journal of Paleolimnology, 49, 513-535.

Englehart, P.J. \& Douglas, A.V. (2005). Changing behavior in the diurnal range of surface air temperatures over Mexico. Geophysical Research Letters, 32, L01701.

Hernández-Morales, R., Ortega-Murillo, M.R., AlvaradoVillanueva, R., Sánchez-Heredia, J.D. \& Medrano-Zarco, F. (2008). Variación anual del fitoplancton en el lago cráter La Alberca de Tacámbaro, Michoacán, México. Biológicas, 10, 5-17.

Hernández-Morales, R., Ortega-Murillo, M.R., SánchezHeredia, J.D., Alvarado-Villanueva, R. \& Aguilera, R. (2011). Distribución estacional del fitoplancton en un lago cálido monomíctico en Michoacán, México. Biológicas, 13, 21-28

Hua, Q., Barbetti, M. \& Rakowski, A.Z. (2013). Atmospheric radiocarbon for the period 1950-2010. Radiocarbon, 55, 2059-2072.

Katz, S.L., Hampton, S.E., Izmest'eva, L.R. \& Moore, M.V. (2011). Influence of long-distance climate teleconnection on seasonality of water temperature in the world's largest lake_Lake Baikal, Siberia. PLoS ONE, 6, 10.

Krammer, K. \& Lange-Bertalot, H. (1986-1991). Subsswasser flora von Mitteleuropa (Teil 1-4). VEB Gustav Fischer Verlag, Stuttgart.

Lewis, W. M. (2000). Basis for the protection and management of tropical lakes. Lakes \& Reservoirs: Research and Management, 5, 35-48.

Ndebele Murisa, M.R., Musil, C.F. \& Raitt, L. (2010). A review of phytoplankton dynamics in tropical african lakes. South African Journal of Science, 106, 13-18.

Pavia, E.G., Graef, F. \& Reyes, J. (2009). Annual and seasonal surface air temperature trends in Mexico. International Journal of Climatology, 29, 1324-1329.

Redfield, A.C. (1958). The biological control of chemical factors in the environment. American Scientist, 64, 205-221.

Reimer, P., Bard, E., Bayliss, A., Beck, W., Blackwell, G., Ramsey, B., Buck, E., Cheng, H., Edwards, L. \& Friedrich, M. (2013). IntCal13 and Marine13 radiocarbon age calibration curves $0-50,000$ years cal BP. Radiocarbon, 55, 1869-1887.

Reynolds, C.S. (1999). Non-determinism to probability, or 
$\mathrm{N}: \mathrm{P}$ in the community ecology of phytoplankton nutrient ratios. Archiv. für Hydrobiologie, 146, 23-35.

Shimoda, Y., Azim, E., Perhar, G., Ramin, M., Kenney, M., Sadraddini, S., Gudimov, A. \& Arhonditsis, G. (2011). Our current understanding of lake ecosystem response to climate change: what have we really learned from the north temperate deep lakes? Journal of Great Lakes Research, 37, 173-193.

Winder, M., Reuter, J.E. \& Schladow, S.G. (2009). Lake warming favours small-sized planktonic diatom species. Proceedings of the Royal Society B: Biological Science, 276, 427-435. 